\title{
Severe Anemia Is Associated with Systemic Inflammation in Young Children Presenting to a Tertiary Hospital in Uganda
}

\author{
Robert O. Opoka, ${ }^{1 \star}$ Andrea L. Conroy, ${ }^{2}$ Ali Waiswa, ${ }^{3}$ Ronald Wasswa, ${ }^{4}$ James K. Tumwine, ${ }^{1}$ Charles Karamagi, ${ }^{1}$ and \\ Chandy C. John ${ }^{2}$ \\ ${ }^{1}$ Department of Paediatrics and Child Health, College of Health Sciences, Makerere University, Kampala, Uganda; ${ }^{2}$ Ryan White Centerfor Pediatric \\ Infectious Disease and Global Health, Indiana University School of Medicine, Indianapolis, Indiana; ${ }^{3}$ Nalufenya Children's Ward, Jinja Regional \\ Referral Hospital, Jinja, Uganda; ${ }^{4}$ Global Health Uganda (GHU) Research Collaboration, Kampala, Uganda
}

\begin{abstract}
The role of inflammation in severe anemia (SA) in African children has not been well characterized. We conducted a study to evaluate risk factors for SA in young children admitted at a tertiary unit in Uganda. Clinical, infectious, and micronutrient risk factors for anemia, along with markers of inflammation, were evaluated in children aged $<5$ years in Jinja Hospital, Uganda. Participants included 284 children with SA (Hemoglobin $[\mathrm{Hb}]<5.0 \mathrm{~g} / \mathrm{dL}$ ), and two control groups: 63 children admitted with acute illness without SA $(\mathrm{Hb}>9.3 \mathrm{~g} / \mathrm{dL})$ and 53 asymptomatic community control children. Appropriate logistic analysis was performed to determine factors associated with SA. Of the 284 children with SA, 36.5\% had Plasmodium falciparum parasitemia, $32.7 \%$ had blackwater fever (one of the types of severe malaria), and $15.5 \% \mathrm{had}$ vitamin B12 deficiency. HIV infection, bacteremia, hookworm infection, severe acute malnutrition, and folate deficiency were relatively uncommon (each accounting for $<8 \%$ ). Factors independently associated with SA compared with the combined control groups included (adjusted odds ratio [OR]; 95\% Cl) the following: $P$. falciparum parasitemia (OR: 4.3; 95\% Cl: 1.4-13.8), total white blood count (OR: 1.3; 95\% Cl: 1.1-1.4), C-reactive protein (OR: 1.8; 95\% Cl: 1.3-2.4), and ferritin (OR: 2.7; 95\% Cl: 1.9-4.0). In this area of Uganda, malaria and markers of inflammation were independently associated with SA in children. Additional studies are required to determine the role of inflammation in children with SA in this population.
\end{abstract}

\section{INTRODUCTION}

Acute episodes of severe anemia (SA) (Hemoglobin $[\mathrm{Hb}] \leq 5$ $\mathrm{g} / \mathrm{dL}$ ) are a public health problem among children living in malaria-endemic areas. They account for $9.7-17.8 \%$ of hospitalizations, with a case fatality rate of $2.7-13.6 \% .{ }^{1,2}$ The causes of SA are multifactorial and interrelated with several of the factors often working together. ${ }^{3}$ However, most studies on SA have evaluated individual etiological factors, ${ }^{4-7}$ and few studies have systematically examined the various etiological factors associated with $S A$ and how these relate to each other. A case-control study of SA in Malawian children identified bacteremia, malaria, hookworm infection, HIV infection, and deficiencies in glucose-6-phosphate dehydrogenase and vitamins $\mathrm{A}$ and $\mathrm{B} 12$ to be the major contributors of SA. ${ }^{8} \mathrm{~A}$ similar study conducted in Papua New Guinea ${ }^{9}$ reported that undernutrition, vitamin A deficiency, infection with parvovirus B19, and infection with Plasmodium falciparum malaria were the main factors associated with SA. These findings suggest that risk factors for SA depend on specific epidemiological settings and environment.

It is postulated that the various etiological factors lead to SA through one of three pathophysiological pathways: hemolysis, acute or chronic blood loss, and dyserythropoiesis. ${ }^{10,11}$ However, one of the few studies that specifically examined the pathophysiology of acute episodes of SA found that irrespective of the etiology, failure of production of red cells because of bone marrow failure was the common final pathway for SA in that population. ${ }^{11}$ The bone marrow failure was postulated to have been mediated by inflammatory mechanisms with downregulation of erythropoietin production. The authors noted that their findings needed to be confirmed in

${ }^{*}$ Address correspondence to Robert O. Opoka, Department of Paediatrics and Child Health, College of Health Sciences, Makerere University, Mulago Hill Road, P.O. Box 7072, Kampala, Uganda. E-mail: opokabob@yahoo.com other settings where etiological factors differ. In Uganda where malarial transmission rates remain high, ${ }^{12}$ the etiological factors associated with SA and the underlying pathological pathways are poorly described. Identification of the etiological factors and pathophysiological pathways involved in SA is necessary to focus context-specific interventions for control and treatment of this common childhood problem.

A number of studies in adults and children show that inflammation plays an important role in anemia, especially in diseases associated with repeated infections, inflammatory disorders, and sepsis. ${ }^{13,14}$ Studies in the critically ill show that inflammation is also an important contributor to anemia of acute illnesses such as severe trauma, myocardial infarction, or sepsis. ${ }^{15}$ In resource-limited settings where infections are common, ${ }^{16}$ inflammatory markers are not well studied as risk factors for SA in children. ${ }^{17}$ Hence, there is need for studies to investigate the role of inflammation alongside the standard clinical, infectious, and micronutrient risk factors for $S A$ in African children.

We evaluated the etiological factors associated with SA in young children presenting to a tertiary unit in Uganda. In addition, we measured erythropoietin and acute inflammatory markers (ferritin and C-reactive protein [CRP]) in the study population to evaluate the association between these biomarkers and SA.

\section{METHODS AND MATERIALS}

Design. This was a comparative cross-sectional study carried out at Jinja Regional Referral (Jinja RR) Hospital in the mideastern part of Uganda. Jinja RR Hospital is a free-forservice public facility that mainly serves the rural population from the Busoga region between the shores of lakes Victoria and Kyoga. The Busoga region is an area of high year-round malaria transmission with a parasite prevalence rate of $24-53 \% .{ }^{18}$ The pediatric ward at Jinja Hospital is an 80 -bed 
unit that admits $30-50$ patients daily. The ward also has a functioning side laboratory able to provide basic microscopy and blood transfusion services.

Study population. We consecutively recruited, at admission, children with $\mathrm{SA}(\mathrm{Hb} \leq 5.0 \mathrm{~g} / \mathrm{dL})$ aged $0-5$ years presenting to the pediatric ward of Jinja RR Hospital. Two comparative groups of children of similar age were enrolled: 1 ) a non-SA $(\mathrm{Hb}>9.3 \mathrm{~g} / \mathrm{dL})$ group of children admitted for acute illnesses without $S A$, and 2) healthy community children (CC) from the family or neighborhoods of the SA children. For all the groups, children who had been transfused in the last 4 weeks or presenting because of surgical conditions or known chronic conditions such as bleeding disorders, malignancy, or sickle cell anemia were excluded. For the healthy $\mathrm{CC}$, children with febrile illnesses at the time of recruitment were excluded. Recruitment of both SA and comparative groups happened concurrently, and matching was performed at the group and not the individual level. Overall, we aimed to recruit about one child in the comparative groups for every two SA cases that were enrolled.

Data and sample collection. A standardized study questionnaire was used to collect data on each child's sociodemographic details, findings on clinical examination, clinical diagnoses, treatment received, results of routine laboratory investigations, and outcome of the hospitalization. In addition, blood, urine, and stool were collected to investigate for known etiological causes of SA. Serum samples were aliquoted and stored at $-80^{\circ} \mathrm{C}$ for future analysis. Study participants were managed as per Ugandan clinical guidelines. ${ }^{19}$ Children with $S A$ received a blood transfusion, if indicated, followed by specific treatment for any comorbid condition identified. All children with evidence of malaria (positive rapid diagnostic test [RDT] or parasites detected by microscopy) were given parenteral artesunate and/or artemether-lumefantrine as per Ugandan guidelines.

Measurements. Height (or length in children younger than 2 years) was measured after recovery using a portable stadiometer, and weight was measured using a digital weighing scale (SECA, Hamburg, Germany). Hemoglobin concentration was measured on-site with a HemoCue system (HemoCue 301, Angelholm, Sweden). The Hb measurement by HemoCue was used to determine eligibility for enrollment and subsequent study group. A complete blood count with differentials was performed using a hematological analyzer at Lancet Laboratories (Beckman Coulter, Brea, CA).

All children had a routine blood smear (Field's stain) and three band ( $P$. falciparum/Pan) rapid diagnostic malaria test (SD Bioline Malaria Ag P. falciparum/Pan, Abbott, Chicago, IL) at admission. In addition, Giemsa-stained thick and thin smears were prepared and read independently by two certified malaria microscopists. Plasmodium falciparum asexual parasites were counted against 200 or 500 white blood cells, and parasite density was calculated based on an estimated total white blood cell count of $8,000 / \mu \mathrm{L}$. Reticulocyte counts were also read manually on new methylene blue $\mathrm{N}$ stained slides by two microscopists. For both malaria and reticulocyte counts, a third reader was required in the event of discordant results or a parasite density or reticulocyte counts that differed by more than $20 \%$. The reticulocyte percent was calculated out of the number of reticulocytes counted in 1,000 red blood cells.

For stool and urine samples, caregivers were provided with clean stool and urine containers to collect a single early morning sample from the study participants. Stool samples were examined for helminths within 1 hour of collection using direct microscopy and iodine preparation. A wet preparation was performed on the fresh urine sample and examined by direct microscopy for Schistosoma haematobium. The microscopy for urine and stool was performed from the side laboratory of the hospital.

Blood culture: Venous blood samples of $1-2 \mathrm{~mL}$ were taken for all children with axillary temperature $>38.0^{\circ} \mathrm{C}$. Samples were incubated in BACTEC 9050 (BD, Franklin Lakes, NJ) automated system for at least 5 days. Mixed growths or coagulasenegative Staphylococcus were considered contaminants.

HIV testing was performed for all children using the rapid diagnostic screening tests (Determine HIV1/2, Abbott, Chicago, IL; HIV1/2 Stat-Pak, Chembio, Medford, NY, and UniGold, Trinity Biotech, Bray, Ireland). Children who were younger than 18 months had their positive HIV screening result confirmed by DNA PCR.

To check for sickle cell genes, $\mathrm{Hb}$ electrophoresis was performed on dried blood spots from the Central Public Health Laboratories in Kampala.

Assays for biomarkers and micronutrients. Laboratory assays were performed on stored serum sample aliquots.

Assessment of vitamin B12 and folic acid. Vitamin B12 and folic acid were measured in serum using microbiological assays (Eagle Biosciences, Amherst, NH). For vitamin B12 assessment, samples (diluted 1:25) and standards were added to microtiter plates coated with Lactobacillus delbrueckii ssp. lactis and incubated at $37^{\circ} \mathrm{C}$ for $44-48$ hours. The amount of vitamin B12 was determined based on the amount of growth of Lactobacillus plantarum measured using turbidimetry at $610 \mathrm{~nm}$ using a SpectraMax M3 multi-mode spectrophotometer (Molecular Devices, San Jose, CA). We tested 10\% of samples in duplicate and had a coefficient of variation of $15.6 \%$ for vitamin B12. The assay range was $2700-75 \mathrm{ng} / \mathrm{L}$. For folic acid, a similar protocol was used, but samples were diluted 1:75 and plated on a microtiter plate coated with Lactobacillus rhamnosus. The assay range was $48-1.5 \mu \mathrm{g} / \mathrm{L}$, and the coefficient of variation for samples tested in duplicate was 3.3\%.

Assessment of CRP and ferritin. C-reactive protein (DuoSet ELISA, R\&D Systems, Minneapolis, MN) and ferritin (Ramco Laboratories, Stafford, TX) were measured in serum by ELISA according to the manufacturer instructions. Dilutions for CRP were $10,000,200,000$, or 250,000 and ferritin were 10,2 , or neat (undiluted). The assay coefficient of variation for CRP and ferritin was $5.3 \%$ and $1.9 \%$, respectively. The assay range for CRP was $0.115-750 \mathrm{mg} / \mathrm{L}$ and for ferritin was $3-40,000 \mathrm{ng} / \mathrm{mL}$.

Definitions. Severe malaria was defined as the presence of asexual forms of $P$. falciparum by microscopy. Recent malarial infection was defined as positive RDT result in the absence of parasitemia on microscopy. Blackwater fever was defined as a history of passage of dark brown, tea, or cola-colored urine during the present episode of illness observed by the caregiver and where possible by the study clinician. To ensure accuracy, color schemes were used to confirm the color of the urine.

Calculations for anthropometric $z$-scores were performed according to WHO growth reference curves with the use of Epi Info 2000 (CDC, Atlanta, GA). Severe acute malnutrition was diagnosed as weight-for-height $z$-scores less than -3 SD or between -2 and -3 in the presence of significant edema. Stunting was defined as a height-for-age $z$-score of less than -2 . 
Vitamin B12 and folate deficiencies were considered present if concentrations were $<200 \mathrm{pg} / \mathrm{mL}$ and $3 \mathrm{ng} / \mathrm{mL}$, respectively. ${ }^{20-22}$ Iron deficiency was defined as ferritin $<12$ $\mu \mathrm{g} / \mathrm{L}$ if CRP was $<10 \mathrm{mg} / \mathrm{L}$ or ferritin $<30 \mu \mathrm{g} / \mathrm{mL}$ if CRP was $\geq 10 \mathrm{mg} / \mathrm{L}$. ${ }^{23}$ Limited maternal education was defined as having spent less than 7 years at school or having no education.

Sample size. We estimated that a sample of 399 children at a ratio of 2:1 between the SA and comparator groups would give us $80 \%$ power to detect a $15 \%$ difference in proportion of children with malaria between the two groups, at 0.05 level of significance.

Statistical analysis. Data were entered in FileMaker Pro and exported to Stata version 14 for analysis (StataCorp., College Station, TX). Descriptive statistics were used to present the data and to determine differences in the study groups. For comparisons, the chi-squared test or Fisher's exact test was used for categorical data, whereas for continuous data, means were compared using the Student's $t$-test and medians using the Wilcoxon rank-sum test. Among the SA children, we determined the frequency of each of the known etiological factors and also determined for each patient the number of associated etiological factors identified. Bivariate logistic regression was used to determine factors associated with SA. In addition to factors known to be associated with SA, that is, age, gender, and maternal education, we also included in the multivariable model factors that were significant $(P<0.05)$ at bivariate analysis. Recent malaria exposure, severe acute malnutrition, and blackwater fever were excluded from the final multivariable model because they were collinear with other variables in the model.

Ethical considerations. Ethical approval was provided by the Makerere University School of Medicine Research and Ethics Committee and the Uganda National Council of
Science and Technology. Written informed consent was obtained from the caregivers of all the children.

\section{RESULTS}

Between June 2016 and January 2018, a total of 417 children were screened and recruited. Seventeen SA children were excluded, one with $\mathrm{Hb}>5.0 \mathrm{~g} / \mathrm{dL}$ and 16 with sickle cell anemia. Four hundred children (SA, $n=284$; non-SA, $n=63$; and $\mathrm{CC}, n=53$ ) were eligible and were included in the analysis. The mean $\mathrm{Hb}$ for SA, non-SA, and CC was 3.6, 11.1, and 11.3 $\mathrm{g} / \mathrm{dL}$, respectively (Table 1 ). There was no difference in the mean age across the three groups. Among the hospitalized children, fever and cough were the most common presenting symptoms (Table 1). Severe anemia children were more likely to be referred, febrile at admission, and had longer mean duration of hospitalization than non-SA children (Table 1). Among the SA children, 153 (53.9\%) had been previously hospitalized with $104(67.9 \%)$ of prior hospitalizations requiring a blood transfusion (Table 1). There were two inpatient deaths in the study, both in the SA group.

Factors associated with SA. Plasmodium falciparum malaria and blackwater fever were the most common diagnoses among SA children, accounting for 103 (36.5\%) and $93(32.7 \%)$, respectively (Table 2$)$. A total of $143(50.4 \%)$ SA children had a recent malaria infection defined as a positive RDT for HRP2 and negative blood slide at the time of admission (Table 2). Asymptomatic parasitemia was found in 5 $(9.4 \%)$ of the CC, whereas $5(7.9 \%)$ of non-SA children had malaria (Table 2). Severe anemia children had higher mean white blood cell counts than non-SA or CC (Table 2). In addition, the levels of markers of acute inflammation (serum ferritin and $\mathrm{CRP}$ ) were significantly higher in the SA children than in

TABLE 1

Demographics, clinical characteristics, and inpatient outcome of study participants

\begin{tabular}{|c|c|c|c|}
\hline & $\mathrm{SA}(N=284)$ & Acute illness, no SA $(N=63)$ & Asymptomatic community children $(N=53)$ \\
\hline Age (years), mean (SD) & $2.3(1.2)$ & $2.1(1.2)$ & $2.3(1.0)$ \\
\hline Gender, male, $n(\%)$ & $179(63.0)$ & $29(46.0)$ & $32(60.4)$ \\
\hline Stunted, $n(\%)^{\star}$ & $74 / 276(26.8)$ & $12(19.1)$ & 19 (35.9) \\
\hline Limited maternal education $\dagger$ & $217(78.1)$ & $29(46.0)$ & 35 (71.4) \\
\hline Axillary temperature $\left({ }^{\circ} \mathrm{C}\right)$, mean $(\mathrm{SD})$ & $37.4(0.8)$ & $36.9(0.9)$ & $36.5(0.4)$ \\
\hline Referred, $n(\%)$ & $172(60.6)$ & $5(7.9)$ & - \\
\hline Immunized up to date, $n(\%)$ & $274(96.5)$ & $61(96.8)$ & - \\
\hline \multicolumn{4}{|l|}{ Presenting features, $n(\%)$} \\
\hline Fever & $280(98.6)$ & $44(69.8)$ & - \\
\hline Cough & $200(70.4)$ & 46 (73.0) & \\
\hline Duration of illness (days), mean (SD) & $3.9(1.8)$ & $3.1(2.4)$ & - \\
\hline \multicolumn{4}{|l|}{ Previous history of, $n$ (\%) } \\
\hline Hospitalization & $153(53.9)$ & $20(31.8)$ & - \\
\hline Transfusion & $104(67.9)$ & $0(0.0)$ & \\
\hline Transfused $>1$ & $57(54.8)$ & $0(0.0)$ & \\
\hline \multicolumn{4}{|l|}{ Clinical presentation, $n(\%)$} \\
\hline Febrile $\left(T>37.4^{\circ} \mathrm{C}\right)$ & $132(46.5)$ & $16(25.4)$ & - \\
\hline Jaundice & $62(21.8)$ & $0(0.0)$ & \\
\hline Hepatomegaly & $71(25.0)$ & $0(0.0)$ & \\
\hline Splenomegaly & 159 (55.9) & $0(0.0)$ & \\
\hline Duration of hospitalization, mean (SD) & $4.4(2.1)$ & $1.5(1.9)$ & - \\
\hline Hemoglobin level (g/dL), mean (SD) & $3.6(0.9)$ & $11.1(1.1)$ & $11.3(1.6)$ \\
\hline Total WBC, mean (SD)‡ & $19.3(13.2)$ & $9.3(3.3)$ & $10.2(3.2)$ \\
\hline Reticulocytosis (> 2.5\%), n (\%) & $50(17.6)$ & $12(19.1)$ & 7/52 (13.5) \\
\hline Died, $n(\%)$ & $2(0.7)$ & $0(0.0)$ & - \\
\hline
\end{tabular}

$\mathrm{SA}=$ severe anemia.

* Stunted $=$ height-for-age $z$-score $<-2$ SD.

† Data available for SA $(n=278)$ and non-SA $(n=49)$.

$\ddagger \mathrm{WBC}=$ white blood count, data available for SA, $n=280$; non-SA, $n=50$; CC, $n=51$. 
TABLE 2

Risk factors for SA in children with SA compared with control groups

\begin{tabular}{|c|c|c|c|c|}
\hline \multirow[b]{2}{*}{ Risk factors, $n(\%)$} & \multirow[b]{2}{*}{$\mathrm{SA}(N=284)$} & \multicolumn{2}{|c|}{ Control groups } & \multirow[b]{2}{*}{$P$-value } \\
\hline & & Non-SA $(N=63)$ & SA vs. controls* & \\
\hline \multicolumn{5}{|l|}{ Clinical } \\
\hline Recent malarial infection & $143(50.4)$ & $10(15.9)$ & $17(32.1)$ & $<0.001$ \\
\hline Severe acute malnutrition & $19(6.7)$ & $0(0.0)$ & $0(0.0)$ & 0.004 \\
\hline \multicolumn{5}{|l|}{ Infection or infection-related } \\
\hline Malaria parasitemia & $103(36.5)$ & $5(7.9)$ & $5(9.4)$ & $<0.001$ \\
\hline Blackwater fever & $93(32.7)$ & $1(1.6)$ & $0 / 53(0)$ & $<0.001$ \\
\hline Hookworm infection & $16 / 222(7.2)$ & $1 / 50(2.0)$ & $2 / 21(9.5)$ & 0.374 \\
\hline HIV infection & $7(2.5)$ & $2(3.2)$ & $0(0)$ & 0.650 \\
\hline Bacteremia & $3 / 54(5.6)$ & $0 / 9(0.0)$ & - & 0.469 \\
\hline \multicolumn{5}{|l|}{ Micronutrient deficiency } \\
\hline Vitamin B12 deficiency & $44(15.5)$ & $6(9.5)$ & - & 0.222 \\
\hline Folate deficiency & $5(1.8)$ & $0(0.0)$ & - & 0.289 \\
\hline \multicolumn{5}{|l|}{ Markers of inflammation } \\
\hline C-reactive proteint (mg/L), mean (SD) & $5.4(1.0)$ & $2.8(2.4)$ & $0.2(1.8)$ & $<0.001$ \\
\hline Ferritin levelł (mg/L), mean (SD) & $8.1(1.7)$ & $4.9(1.6)$ & $4.1(1.4)$ & $<0.001$ \\
\hline
\end{tabular}

children in the control groups (Table 2). There was, however, no statistically significant difference in the proportion of children with high reticulocyte count in the three groups (Table 2).

Micronutrient deficiencies. There was no difference in the prevalence of vitamin B12 and folate deficiencies between children with SA and those in the control groups. Iron deficiency could not be accurately measured because many children had very elevated ferritin and CRP levels, suggesting inflammation that altered ability to use ferritin as a marker of iron deficiency.

Infections. The prevalence of HIV infection was 7 (2.7\%) in SA, $2(3.2 \%)$ in non-SA, and 0/53 (0.0\%) in CC (Table 2). A blood culture was performed in a total of 54 SA children, of which $3(5.6 \%)$ were positive. The invasive bacteria species identified were Citrobacter, Providencia, and Staphylococcus aureus. Stool samples were available for 293 (73.3\%) study participants, and hookworm ova were seen in 16 (7.2\%) children with SA. The prevalence of hookworm infection was not different between children with SA and the control groups (Table 2). Urine samples were collected from 287 participants, including 234 (77.7\%) children with SA. However, none of the samples were positive for $S$. haematobium.

Overall, nearly half of the children had an associated etiological factor identified for SA ( $n=128,45.1 \%)$, whereas 69 $(24.3 \%)$ children had multiple associated etiological factors identified. The majority $(70 / 87 ; 80.1 \%)$ of the SA children with no associated etiological factors identified had recent malarial infection.

Regression analysis of independent factors associated with $S A$. To determine the etiological factors and biomarkers associated with SA, we used logistic regression. The final model compared the factors between children with SA and those in the control groups (non-SA and CC) combined. We found the factors associated with SA were malaria parasitemia (OR: 4.3; 95\% Cl: 1.4-13.8), unit increase in total white blood count (OR: 1.3; 95\% Cl: 1.1-1.4), CRP (OR: 1.8; 95\% Cl: 1.3-2.4), and ferritin (OR: 2.7; 95\% Cl: 1.9-4.0) (Table 3). These findings were similar even when children with SA were compared separately with either of the control groups (non-SA or CC).

\section{DISCUSSION}

We evaluated the etiological factors and biomarkers of host response in hospitalized children with SA in Jinja RR hospital. We identified multiple etiological factors associated with SA, which is consistent with results from other studies in malaria-endemic areas., 8,24 In this study, like others in malaria-endemic areas, $P$. falciparum was an important contributor to SA in young children. The prevalence of malaria parasitemia was $36.5 \%$ in children with SA and was lower than the $54 \%$ reported in earlier studies from similar settings. ${ }^{1}$ In this study, we found that SA children had markedly elevated levels of ferritin and CRP (markers of host response) compared with control children.

Malaria remains a significant contributor to SA. In this study, an additional $50.5 \%$ of the children with SA, although malaria smear negative by microscopy, had recent exposure to malaria based on a positive RDT test. This suggests that malaria exposure in the community is still high despite the ongoing malaria control interventions being implemented. ${ }^{25}$ The high rate of RDT-positive but smear-negative malaria may be due to the availability and easy access to effective antimalarials in the community that rapidly clear parasitemia before presentation to hospital. It is also probable that some of the RDT-positive malaria was due to submicroscopic parasitemia, a phenomenon that is increasingly being recognized in malaria-endemic areas. ${ }^{26,27}$ Irrespective of the reasons for the negative smear, RDT-positive malaria is likely a significant contributor to the high burden of SA found in this community. It is possible that these smear-negative children present before making a complete recovery from malaria-related anemia of the recent malarial episode. It is also possible that the anemia may be due to post-artesunate delayed hemolysis that has been reported in some children. ${ }^{28-30}$ Taken together, our findings support the assertion in malarial-endemic areas that it is exposure rather than the level of parasitemia that is important in the development of SA. ${ }^{26,31,32}$ Efforts to reduce SA in children living in malaria-endemic areas should therefore focus on preventing exposure to malaria. 
TABLE 3

Etiological factors associated with severe anemia in children younger than 5 years presenting to Jinja Regional Referral Hospital

\begin{tabular}{|c|c|c|c|c|}
\hline & \multicolumn{2}{|c|}{ Unadjusted } & \multicolumn{2}{|c|}{ Adjusted $^{*}$} \\
\hline & OR $(95 \% \mathrm{Cl})$ & $P$-value & OR $(95 \% \mathrm{Cl})$ & $P$-value \\
\hline Malaria parasitemia & $6.0(3.0,12.0)$ & $<0.001$ & $4.4(1.4,13.8)$ & 0.012 \\
\hline Total white blood count & $1.3(1.2,1.3)$ & $<0.001$ & $1.3(1.1,1.4)$ & $<0.001$ \\
\hline C-reactive proteins (mg/L) & $2.9(2.3,3.6)$ & $<0.001$ & $1.8(1.3,2.4)$ & $<0.001$ \\
\hline Ferritin (mg/L) & $4.1(3.0,5.6)$ & $<0.001$ & $2.7(1.9,4.0)$ & $<0.001$ \\
\hline Age & $1.1(0.95,1.4)$ & 0.170 & - & - \\
\hline Gender & $0.65(0.4,1.0)$ & 0.054 & - & - \\
\hline Vitamin B12 deficiency & $1.7(0.7,4.3)$ & 0.227 & - & - \\
\hline Hookworm infection & $3.8(0.5,29.4)$ & 0.200 & - & - \\
\hline HIV infection & $1.6(0.3,7.0)$ & 0.652 & - & - \\
\hline
\end{tabular}

${ }^{\star}$ Also adjusted for age, gender, and maternal education.

Blackwater fever, a form of severe malaria, was one of the most prevalent etiological factors of SA identified in this study. Blackwater fever is common in eastern Uganda and presents in up to $21 \%$ of critically ill hospitalized children. ${ }^{33,34}$ Children who develop this condition are prone to recurrent episodes of SA requiring repeated blood transfusions. The etiology of blackwater fever and trigger for SA in this condition are not known. In this study, the prevalence of blackwater fever was similar in children with recent malarial infections and those with smear-positive malaria. So, it is unlikely to be a marker of recent malarial infection. Blackwater fever was also not an independent predictor of SA as it was correlated to malaria parasitemia. It is however possible that blackwater fever may be a marker of more significant hemolysis that might be genetic or related to parasitemia. Further research is needed to establish the pathophysiology and treatment of this condition.

Other etiological factors were relatively uncommon in this study. The HIV prevalence rate in the study was lower than the $12.6 \%$ reported in the Malawian study ${ }^{8}$ but similar to recent HIV rates reported in hospitalized children in East Africa. ${ }^{33,35}$ The prevalence of bacteremia in this study was also lower than that in the Malawian study where $15 \%$ of SA children were culture positive, ${ }^{8}$ but similar to what has been reported in malarial studies in similar settings. ${ }^{9,35}$ An earlier study conducted at Jinja RR Hospital reported a bacteremia rate of $19 \%$ among febrile young children without parasitemia. ${ }^{36}$ However, that study was conducted before the introduction of pneumococcal conjugate vaccine for routine childhood immunization in Uganda. There is presently an ongoing surveillance study to determine the impact of this new vaccination on childhood inpatient morbidity.

The prevalence of undernutrition, and folate and vitamin B12 deficiencies was relatively low in the study population and did not differ between groups. We were unable to make conclusions on the level of iron deficiency in this study because of the high level of inflammation found in the study patients. The role of iron deficiency in the etiology of SA however remains unclear, with some studies finding it protective ${ }^{8}$ and others not protective, ${ }^{9}$ whereas in others, it was a significant risk factor for SA. ${ }^{24}$ The Papua New Guinea study also found that vitamin A deficiency and hookworm infection were associated with $\mathrm{SA},{ }^{9}$ although hookworm infection was not associated with SA in this study. The relatively low prevalence of hookworm infection is likely attributable to the national deworming program in Uganda where all children younger than 5 years are dewormed every 6 months.

In this study, we found that SA was associated with the biomarkers of host response as seen in the markedly raised levels of CRP and ferritin. The high levels of these biomarkers of host response found in this study support findings from previous studies of the central role that inflammation plays in the pathogenesis of SA, irrespective of the etiology, in malariaendemic areas. ${ }^{37}$ However, the pathogenic pathways responsible for the development of SA remain unclear. Studies on the pathogenesis of severe malarial anemia suggest that anemia mainly results from the suppression of erythropoietininduced erythropoiesis by parasite factors, such as hemozoin, ${ }^{38}$ mediated by inflammatory cytokines. ${ }^{10}$ In Malawian children, it was found that irrespective of the etiological factor, suppression of erythropoiesis leading to red cell production failure was the predominant pathway for the development of SA. ${ }^{11}$ Interestingly, we found no significant difference in reticulocyte count across groups. This suggests a failure of the bone marrow in the SA group, given the profound anemia this group. It is likely that insufficient iron delivery to the bone marrow to support new red cell formation happens in the context of anemia of inflammation with iron sequestration and decreased iron uptake in the gut due to hepcidin binding and degrading gut ferroportin. Overall, our finding of the strong association between the markers of inflammation and SA highlights the central role of inflammatory mechanisms in the pathways leading to SA.

The levels of ferritin and CRP differed between the different forms of SA children. Ferritin levels were highest in SA children without malaria, whereas CRP was highest in the group with parasitemia and lowest in SA children without malarial infection. Taken together, these findings suggest that the inflammatory markers involved and the mechanisms of suppression on red cell production may be different for malarial and non-malarial causes of SA. Further research is needed to understand the mechanisms by which inflammation mediates dyserythropoiesis for the various etiologies of SA.

Severe anemia children also had significantly higher mean total white blood cell counts than children in the control groups. Among SA children, the levels of white blood cell counts were significantly elevated in children with recent malarial infection and no malaria than in children with parasitemia. This may be due to the strong association between recent malaria exposure and increased risk of Gram-negative bacteremia. ${ }^{39,40}$ Although the prevalence of bacteremia was low in this study, the elevated levels of white blood cell count may be markers of infection in children without parasitemia. Children with recent malarial infection had higher $(8.7 \%)$ levels of bacteremia than those with parasitemia (3.7\%), although the difference was not statistically significant.

The inpatient mortality in this study was relatively low, confirming findings that with SA, prompt availability of blood 
transfusion services is the critical factor in inpatient mortality. ${ }^{41}$ However, more than a third $(36.7 \%)$ of the SA children had a history of previous transfusion, suggesting that SA is a recurrent problem in these children. Thus, there is need for longitudinal studies to identify SA children at risk of poor postdischarge outcomes.

Our study had some limitations. We were not able to evaluate for all possible known causes of SA. For example, we did not do viral or genetic studies, so possible viral and genetic factors were not documented. The biomarkers of host response were measured once, and so we were not able to establish the temporal relationship with the development of SA. In addition, we were unable to do bone marrow biopsies to establish the functionality of the bone marrow for the various etiologies of SA. However, we evaluated for the most common available causes of SA in our setting.

In summary, we identified multiple etiological factors associated with SA in young children presenting to Jinja Hospital. Plasmodium falciparum malaria and blackwater fever were the most prevalent etiological diagnoses in SA children. In multivariable analysis, markers of host responses, that is, ferritin and CRP, were significantly associated with SA. There is need for studies to confirm the immunopathogenic pathways of SA in malaria-endemic areas to target intervention strategies.

Received March 17, 2020. Accepted for publication July 29, 2020.

Published online September 8, 2020.

Acknowledgments: We thank Jinja Regional Referral Hospital and Global Health Uganda (GHU) for the permission to conduct this study in their facilities, all staff at these centers for their contribution to this work, and Gloria Kyarisiima, Gorretti Kemigisha, and Aliwuya Sadat for their dedication in collecting and compiling the data.

Financial support: This work was supported by funding from D43 grants from the National Institutes of Health (NURTURE, grant number D43TW010132), the Fogarty International Center (grant number D43 NS078280), and the Research Council of Norway through the Global Health and Vaccination Programme (GLOBVAC), project number 234487.

Authors' addresses: Robert O. Opoka, Department of Paediatrics, Makerere University, Kampala, Uganda, E-mail: opokabob@yahoo.com. Andrea L. Conroy, Department of Medicine, University of Toronto, Toronto, Canada, E-mail: andrea.conroy@gmail.com. Ali Waiswa, Department of Paediatrics, Jinja Regional Referral Hospital, Paediatrics, Jinja, Uganda, E-mail: aliwaiswabaligeya@gmail.com. Ronald Wasswa, Laboratory, Global Health Uganda, Kampala, Uganda, E-mail: wasswar@gmail.com. James K. Tumwine, Department of Pediatrics and Child Health, College of Health Sciences, Makerere University, Kampala, Uganda, E-mail: kabaleimc@gmail.com. Charles Karamagi, Department of Paediatrics and Child Health, College of Health Sciences, Makerere University, Kampala, Uganda, and Clinical Epidemiology Unit, College of Health Sciences, Makerere University, Kampala, Uganda, E-mail: ckaramagi2000@yahoo.com. Chandy C. John, Department of Paediatrics, Ryan White Center for Pediatric Infectious Disease and Global Health, Indiana University, Indianapolis, IN, E-mail: chjohn@iu.edu.

This is an open-access article distributed under the terms of the Creative Commons Attribution (CC-BY) License, which permits unrestricted use, distribution, and reproduction in any medium, provided the original author and source are credited.

\section{REFERENCES}

1. Kiggundu VL et al., 2013. High prevalence of malaria parasitemia and anemia among hospitalized children in Rakai, Uganda. PLoS One 8: e82455.
2. Muoneke VU, Ibekwe RC, Nebe-Agumadu HU, Ibe BC, 2012. Factors associated with mortality in under-five children with severe anemia in Ebonyi, Nigeria. Indian Pediatr 49: 119-123.

3. Brabin BJ, Premji Z, Verhoeff F, 2001. An analysis of anemia and child mortality. J Nutr 131: 636S-645S.

4. Koram KA, Owusu-Agyei S, Utz G, Binka FN, Baird JK, Hoffman SL, Nkrumah FK, 2000. Severe anemia in young children after high and low malaria transmission seasons in the KassenaNankana district of northern Ghana. Am J Trop Med Hyg 62: 670-674.

5. Malamba S, Hladik W, Reingold A, Banage F, McFarland W, Rutherford G, Mimbe D, Nzaro E, Downing R, Mermin J, 2007. The effect of HIV on morbidity and mortality in children with severe malarial anaemia. Malar J 6: 143.

6. Novelli EM, Hittner JB, Davenport GC, Ouma C, Were T, Obaro S, Kaplan S, Ong'echa JM, Perkins DJ, 2010. Clinical predictors of severe malarial anaemia in a holoendemic Plasmodium falciparum transmission area. Br J Haematol 149: 711-721.

7. Nyesigire Ruhinda E, Bajunirwe F, Kiwanuka J, 2012. Anaemia in HIV-infected children: severity, types and effect on response to HAART. BMC Pediatr 12: 170

8. Calis JC et al., 2008. Severe anemia in Malawian children. NEngl $J$ Med 358: 888-899.

9. Manning L, Laman M, Rosanas-Urgell A, Michon P, Aipit S, Bona C, Siba P, Mueller I, Davis TM, 2012. Severe anemia in Papua New Guinean children from a malaria-endemic area: a casecontrol etiologic study. PLoS Negl Trop Dis 6: e1972.

10. Perkins DJ, Were T, Davenport GC, Kempaiah P, Hittner JB, Ong'echa JM, 2011. Severe malarial anemia: innate immunity and pathogenesis. Int J Biol Sci 7: 1427-1442.

11. Boele van Hensbroek M, Calis JC, Phiri KS, Vet R, Munthali F, Kraaijenhagen $\mathrm{R}$, van den Berg $\mathrm{H}$, Faragher B, Bates I, Molyneux ME, 2010. Pathophysiological mechanisms of severe anaemia in Malawian children. PLoS One 5: e12589.

12. Okiro EA, Bitira D, Mbabazi G, Mpimbaza A, Alegana VA, Talisuna AO, Snow RW, 2011. Increasing malaria hospital admissions in Uganda between 1999 and 2009. BMC Med 9: 37.

13. Nemeth $E$, Ganz T, 2014. Anemia of inflammation. Hematol Oncol Clin North Am 28: 671-681.

14. Weiss G, Ganz T, Goodnough LT, 2019. Anemia of inflammation. Blood 133: 40-50.

15. Sihler KC, Napolitano LM, 2008. Anemia of inflammation in critically ill patients. J Intensive Care Med 23: 295-302.

16. Engle-Stone R et al., 2017. Predictors of anemia in preschool children: biomarkers reflecting inflammation and nutritional determinants of anemia (BRINDA) project. Am J Clin Nutr 106: 402s-415s.

17. Foote EM, Sullivan KM, Ruth LJ, Oremo J, Sadumah I, Williams TN, Suchdev PS, 2013. Determinants of anemia among preschool children in rural, western Kenya. Am J Trop Med Hyg 88: 757-764.

18. Uganda Bureau of Statistics (UBOS), 2018. Uganda Demographic and Health Survey 2016. Kampala, Uganda and Rockville, MA: Uganda Bureau of Statistic and ICF.

19. Ministry of Health Uganda, 2016. Uganda Clinical Guidelines 2016. Kampala, Uganda: Ministry of Health Uganda.

20. Devalia V, Hamilton MS, Molloy AM, 2014. Guidelines for the diagnosis and treatment of cobalamin and folate disorders. Br J Haematol 166: 496-513.

21. Matchar DB, McCrory DC, Millington DS, Feussner JR, 1994. Performance of the serum cobalamin assay for diagnosis of cobalamin deficiency. Am J Med Sci 308: 276-283.

22. Snow CF, 1999. Laboratory diagnosis of vitamin B12 and folate deficiency: a guide for the primary care physician. Arch Intern Med 159: 1289-1298.

23. WHO, 2011. Serum Ferritin Concentrations for the Assessment of Iron Status and Iron Deficiency in populations. Vitamin and Mineral Nutrition Information System. Geneva, Switzerland: World Health Organization.

24. Moraleda $\mathrm{C}$ et al., 2017. Anaemia in hospitalised preschool children from a rural area in Mozambique: a case control study in search for aetiological agents. BMC Pediatr 17: 63.

25. Ssempiira J, Nambuusi B, Kissa J, Agaba B, Makumbi F, Kasasa $S$, Vounatsou $P, 2017$. The contribution of malaria control 
interventions on spatio-temporal changes of parasitaemia risk in Uganda during 2009-2014. Parasit Vectors 10: 450.

26. Katrak S, Nayebare P, Rek J, Arinaitwe E, Nankabirwa JI, Kamya M, Dorsey G, Rosenthal PJ, Greenhouse B, 2018. Clinical consequences of submicroscopic malaria parasitaemia in Uganda. Malar J 17: 67.

27. Rek J et al., 2016. Characterizing microscopic and submicroscopic malaria parasitaemia at three sites with varied transmission intensity in Uganda. Malar J 15: 470.

28. Burri C, Ferrari G, Ntuku HM, Kitoto AT, Duparc S, Hugo P, Mitembo DK, Lengeler C, 2014. Delayed anemia after treatment with injectable artesunate in the Democratic Republic of the Congo: a manageable issue. Am J Trop Med Hyg 91: 821-823.

29. Jauréguiberry S et al., 2014. Postartesunate delayed hemolysis is a predictable event related to the lifesaving effect of artemisinins. Blood 124: 167-175.

30. Rolling $T$ et al., 2014. Delayed hemolysis after treatment with parenteral artesunate in African children with severe malaria-a double-center prospective study. J Infect Dis 209: 1921-1928.

31. Safeukui I et al., 2015. Malaria induces anemia through CD8+ T cell-dependent parasite clearance and erythrocyte removal in the spleen. MBio 6: e02493-14.

32. Idro R, Aloyo J, Mayende L, Bitarakwate E, John CC, Kivumbi GW, 2006. Severe malaria in children in areas with low, moderate and high transmission intensity in Uganda. Trop Med Int Health 11: $115-124$

33. Maitland $\mathrm{K}$ et al., 2011. Mortality after fluid bolus in African children with severe infection. N Engl J Med 364: 2483-2495.
34. Olupot-Olupot $P$ et al., 2017. High frequency of blackwater fever among children presenting to hospital with severe febrile illnesses in eastern Uganda. Clin Infect Dis 64: 939-946.

35. Bangirana P, Opoka RO, Boivin MJ, Idro R, Hodges JS, Romero RA, Shapiro E, John CC, 2014. Severe malarial anemia is associated with long-term neurocognitive impairment. Clin Infect Dis 59: 336-344.

36. Kibuuka A, Byakika-Kibwika P, Achan J, Yeka A, Nalyazi JN, Mpimbaza A, Rosenthal PJ, Kamya MR, 2015. Bacteremia among febrile Ugandan children treated with antimalarials despite a negative malaria test. Am J Trop Med Hyg 93: 276-280.

37. Namaste SM et al., 2017. Adjusting ferritin concentrations for inflammation: biomarkers reflecting inflammation and nutritional determinants of anemia (BRINDA) project. Am J Clin Nutr 106: 359s-371s.

38. Thawani N, Tam M, Bellemare MJ, Bohle DS, Olivier M, de Souza JB, Stevenson MM, 2014. Plasmodium products contribute to severe malarial anemia by inhibiting erythropoietin-induced proliferation of erythroid precursors. J Infect Dis 209: 140-149.

39. Davenport GC et al., 2016. Reduced parasite burden in children with falciparum malaria and bacteremia coinfections: role of mediators of inflammation. Mediators Inflamm 2016: 4286576.

40. Were T, Davenport GC, Hittner JB, Ouma C, Vulule JM, Ong'echa JM, Perkins DJ, 2011. Bacteremia in Kenyan children presenting with malaria. J Clin Microbiol 49: 671-676.

41. English M, Ahmed M, Ngando C, Berkley J, Ross A, 2002. Blood transfusion for severe anaemia in children in a Kenyan hospital. Lancet 359: 494-495. 